\title{
Characterization of Extended-Spectrum
}

\section{$\beta$-Lactamase-Producing Uropathogenic Escherichia coli Among Iranian Kidney Transplant Patients}

This article was published in the following Dove Press journal:

Infection and Drug Resistance

\author{
Mehrdad Halaji (D) ${ }^{1,2}$ \\ Shahrzad Shahidi ${ }^{3}$ \\ Abdolamir Atapour ${ }^{3}$ \\ Behrooz Ataei ${ }^{4}$ \\ Awat Feizi $\mathbb{1 D}^{5}$ \\ Seyed Asghar Havaei ${ }^{1,4}$
}

'Department of Microbiology, School of Medicine, Isfahan University of Medical

Sciences, Isfahan, Iran; ${ }^{2}$ Nosocomial Infection Research Center, Isfahan University of Medical Sciences, Isfahan, Iran; ${ }^{3}$ ssfahan Kidney Diseases Research Center, Isfahan University of Medical Sciences, Isfahan, Iran; ${ }^{4}$ Infectious

Diseases and Tropical Medicine Research Center, Isfahan University of Medical Sciences, Isfahan, Iran; ${ }^{5}$ Department of Biostatistics and Epidemiology, School of Health, Isfahan University of Medical

Sciences, Isfahan, Iran
Correspondence: Seyed Asghar Havaei Infectious Diseases and Tropical Medicine Research Center, Isfahan University of Medical Sciences, Isfahan, Iran

$\mathrm{Tel}+983137922478$

Email havaei@med.mui.ac.ir
Introduction: The aim of this study was to investigate the antimicrobial susceptibility pattern and the presence of ESBLs among the uropathogenic Escherichia coli (UPEC) isolated from kidney transplant patients $(\mathrm{KTP})$ and community-acquired urinary tract infections (UTIs) using phenotypic and molecular methods.

Materials and Methods: A total of 111 pure cultures of UPEC isolates were collected from 65 and 46 of non-KTP and KTPs with UTIs. The pattern and ESBL production of the strains were evaluated. PCR reaction to detect the presence of $b l a_{\mathrm{SHV}}, b l a_{\mathrm{TEM}}$, and $b l a_{\mathrm{CTX}-\mathrm{M}}$ genes was performed.

Results: The results revealed that most of UPEC isolates obtained from KTPs and control group were resistant to trimethoprim/sulfamethoxazole ( $84.8 \%$ vs $46.2 \%$ ), while carbapenems (100\% sensitivity) were the most effective against UPEC isolates. ESBL-producing strains were significantly more frequent in KTPs compared with control group $(43.5 \% \mathrm{vs}$ $23.1 \%, P=0.021)$. The molecular results revealed that 53.2\% (59/111), 45\% (50/111), and $5.4 \%(6 / 111)$ of isolates harbored $b l a_{\mathrm{CTX}-\mathrm{M}}, b l a_{\mathrm{TEM}}$, and $b l a_{\mathrm{SHV}}$ genes, respectively. Of the genes investigated, $b l a_{\text {СТХ-M }}$ and $b l a_{\text {TEM }}$ genes were significantly higher among KTP than the control group.

Conclusion: Our results showed a high proportion of multidrug-resistant and ESBL-producing isolates, which most of them harbor blaCTX-M. A significant high co-resistance to different classes of antibiotics was reported from ESBL-producing UPEC from KTPs, which remains a serious clinical challenge.

Keywords: UTIs, ESBL, PCR, KTP

\section{Introduction}

Bacterial urinary tract infections (UTIs) are the most common infectious complications among kidney transplant patients (KTPs) with diseases ranging from asymptomatic bacteriuria $(\mathrm{AB})$ to potentially life-threatening acute such as cystitis and pyelonephritis. ${ }^{1}$ In most cases, the first year following transplantation is the period of the highest risk for UTI and recurrent infections occur in up to $72 \%$ of kidney transplant (KT) recipients. ${ }^{2,3}$

Female sex, longer duration of catheterization, immunosuppression, diabetes mellitus, and manipulation of the urinary tract are the most important risk factors for UTI after kidney transplantation (KTx). ${ }^{2}$ Previous studies indicated that uropathogenic Escherichia coli (UPEC) is the major causative agent of UTIs after $\mathrm{KTx}^{4}$ Although UTIs should be controlled using antimicrobial therapy, the 
increased rate of antimicrobial resistance has become a major concern causing the emergence of multiple drug resistance in UPEC, especially among KTPs. ${ }^{5,6}$

Antimicrobial resistance leads to higher medical costs, prolonged hospital stay, and increased mortality. This outcome is excessive and inappropriate use of antibiotics, the default in treatment, poor infection prevention and control, and microbial characteristics. ${ }^{7,8}$ UTIs caused by extendedspectrum $\beta$-lactamase (ESBL)-producing Escherichia coli account for one of the major therapeutic and epidemiologic challenges in KTPs. ${ }^{9}$ The prevalence of infections caused by ESBL-producing $E$. coli has steadily been increased in recent years that significantly reduces therapeutic options and is followed by an increased mortality rate. ESBLproducing bacteria can hydrolyze and confer resistance to cephalosporins, penicillins, and monobactams and are inhibited by clavulanic acid. Genes encoding ESBL are often located on transferrable bacterial plasmid DNA. ESBL genes have also been established within integronlike structures. ${ }^{10}$ Furthermore, $\beta$-lactamases may be chromosomally encoded and universally present in a species. ${ }^{3,11}$ The plasmid-mediated $\beta$-lactamase in gram-negative bacteria contains TEM, SHV, and CTX-M enzymes. ${ }^{3,12}$ Among different plasmid-mediated $\beta$-lactamases, several reports suggest that CTX-M-type ESBLs may now be the most predominant ESBL type worldwide. ${ }^{13}$ Owing to the high rate of UTIs affecting renal transplantation and outcomes of ESBL phenotype, it is necessary to assess the weight and control the spread of ESBLs producing isolates among KTPs. ${ }^{14}$ In this study, we aimed to investigate the antimicrobial susceptibility pattern and the presence of ESBLs among the UPESs isolated from KTPs and non-KTPs with UTIs using phenotypic and molecular methods.

\section{Materials and Methods}

\section{Study Population and Bacterial Isolates and Identification}

A case-control study was performed on a collection of 65 nonduplicate UPEC isolates obtained from non-KTP with UTIs, as control group and 46 non-repetitive UPEC isolates obtained from KTPs with UTIs as a case-group, from June 2019 to October 2019. Data were collected from non-KTP who referred to the three laboratory center and KTP were referred from two nephrology private clinic. After screening of the electronic records of the patients, repetitive samples of patients and isolates obtained from the non-KTP group with a history of antibiotic usage in the previous 2 weeks were excluded from the study. This study was evaluated and approved by the Ethics Committee of Isfahan University of Medical Sciences (IR.MUI.MED.REC.1398.202). Moreover, all participants provided written informed consent.

All UPEC isolates were identified using standard microbiological tests such as Gram-staining and biochemical testing (oxidase, sugar fermentation, IMViC, Kliger's iron agar, nitrate reduction, motility, etc.). To confirm the species, we carried out a polymerase chain reaction (PCR) detection based on the uid gene for $\beta$-glucuronidase of E. coli. ${ }^{15}$ Confirmed E. coli isolates were preserved at $-80^{\circ} \mathrm{C}$ for further evaluation.

\section{Antimicrobial Susceptibility Testing}

Antibiotic susceptibility pattern was performed on MullerHinton agar (HiMedia Co., India) using the disk diffusion method, as recommended by the Clinical and Laboratory Standards Institute (CLSI). ${ }^{16}$

For this assay, 16 antibiotics (BD BBL ${ }^{\text {TM }}$ Sensi-Disc ${ }^{\mathrm{TM}}$ ) including amoxicillin/clavulanic acid, piperacillin/tazobactam, cefoxitin, ceftazidime, cefepime, cefixim, imipenem, meropenem, trimethoprim/sulfamethoxazole, ciprofloxacin, ofloxacin, nalidixic acid, norfloxacin, nitrofurantoin, gentamicin, amikacin were used. E. coli ATCC 25922 was utilized as the quality control strain for antibacterial susceptibility testing. ${ }^{16}$ The isolates non-susceptible to $\geq 1$ agent in $\geq 3$ different antimicrobial categories were considered as MDR. ${ }^{17}$

\section{ESBLs Screening Test}

ESBL production was detected using the double-disk synergy test (cefotaxime $(30 \mu \mathrm{g})$ and ceftazidime $(30 \mu \mathrm{g})$ ) alone and in combination with clavulanic acid $(10 \mu \mathrm{g})$ based on CLSI recommendations. Briefly, the phenotypic confirmatory test was performed by comparing the inhibition zone of disks containing cefotaxime and ceftazidime with and without clavulanic acid. After overnight incubation at $37^{\circ} \mathrm{C}$, an increase in the inhibition zone diameter of $\geq 5 \mathrm{~mm}$ between the single disk and the double disk was confirmed as ESBL production. Klebsiella pneumoniae ATCC 700603 (Positive control) and E. coli ATCC 25922 (Negative control) were used as control strains in accordance with CLSI guidelines. ${ }^{16}$

\section{Molecular Characterization of $\beta$-Lactamases}

Genomic DNA was extracted from fresh colonies as described previously. To detect antibiotic resistance genes including $b l a_{\mathrm{SHV}}, b l a_{\mathrm{CTX}-\mathrm{M}}, b l a_{\mathrm{TEM}}-\mathrm{F}$ (AGTATTCAAC ATTTCCGTGTC), and bla ${ }_{\text {TEM }^{-R}}$ (GCTTAATCAGTGA 
GGCACCTATC), separate PCR reactions were performed as previously described. ${ }^{18,19}$

PCR was performed using commercially available PCR Master Mix (AMPLIQON, Denmark) based on the manufacturer's instructions. Briefly, $6 \mu \mathrm{L}$ of Master Mix, $0.3 \mu \mathrm{L}$ of each primer (10 pmoles $/ \mu \mathrm{L})$, and $4.4 \mu \mathrm{L}$ DNase-free distilled water and finally, $1 \mu \mathrm{L}$ template DNA was added in a final volume of $12.5 \mu \mathrm{L}$. PCR amplifications of genes were performed with an initial denaturation step at $94^{\circ} \mathrm{C}$ for $5 \mathrm{~min}$, followed by 30 cycles of denaturation at $94^{\circ} \mathrm{C}$ for $30 \mathrm{~s}$, annealing for 55-57 s at primer-specific temperatures, extension at $72^{\circ} \mathrm{C}$ for $30 \mathrm{~min}$, and a final extension step at $72^{\circ} \mathrm{C}$ for $5 \mathrm{~min}$. The PCR-products were separated by electrophoresis in 1\% agarose gels with $1 \mathrm{X}$ TAE (Tris/ Acetate/EDTA) buffer, stained with safe stain load dye (CinnaGen Co., Iran) and visualized under ultraviolet illumination.

\section{Statically Analysis}

Statistical analysis was carried out through the SPSS ${ }^{\mathrm{TM}}$ software, version 16 (IBM Corp., USA). Categorical variables were expressed as counts and percentages. The Fisher's exact or Chi-square $(\chi 2)$ tests were used to analyze significant differences. A $P$-value $<0.05$ was considered as statistically significant.

\section{Results}

In this study, a total of 111 confirmed UPEC were isolated, $69.6 \%(32 / 46)$ and $30.4 \%(14 / 46)$ isolates were collected from female and male KTP patients, while $69.2 \%(45 / 65)$ and $30.8(20 / 65)$ isolates were obtained from female and male control group, respectively. Demographic and characterization of KTP and non-KTP are summarized in Table 1.

\section{Antibiotic Susceptibility Pattern of UPEC Isolates}

The results of antibiotic susceptibility pattern revealed that more than $52 \%$ of UPEC strain isolated from KTP were resistant to trimethoprim/sulfamethoxazole (84.8\%), nalidixic acid (78.3\%), cefixim (58.7\%), ciprofloxacin $(54.3 \%)$, norfloxacin $(56.5 \%)$, and ofloxacin $(52.2 \%)$, while carbapenems $(100 \%)$, amikacin $(100 \%)$, and nitrofurantoin $(71.7 \%)$ were most effective in vitro antibiotics against UPEC isolates.

Antibiotic susceptibility pattern on the control group revealed that the highest resistance rate was to nalidixic
Table I Distribution of Characteristics of Kidney Transplant Recipients

\begin{tabular}{|l|l|l|}
\hline Characterization & $\begin{array}{l}\text { KTx } \\
\text { Recipients* } \\
\text { n (\%) }\end{array}$ & $\begin{array}{l}\text { Non-KTP } \\
\text { n (\%) }\end{array}$ \\
\hline Sex (female/male) & $32 / 14$ & $45 / 20$ \\
Age (Mean, Median, Range) & $50.8 ; 53 ;(15-82)$ & $45: 42 ;(I-80)$ \\
Hypertension & $50 \%(19 / 38)$ & - \\
Diabetes mellitus & $28.9 \%(1 / / 38)$ & $7.7 \%(5 / 65)$ \\
Immunosuppression & $100(46 / 46)$ & $0(0 / 65)$ \\
Time from last KTx to UTI & 4 month- & - \\
(Range) & 21 year & \\
Ureteral stent or urinary catheter & $0(0 / 38)$ & $0(0 / 65)$ \\
Hospitalization & $0(0 / 46)$ & $0(0 / 65)$ \\
Recurrent UTI & $42.1 \%(16 / 38)$ & - \\
History of UTI & $57.9 \%(22 / 38)$ & - \\
Antibiotic therapy & $31.6 \%(12 / 38)$ & $0(0 / 65)$ \\
\hline Clinical data of a three-month & & \\
period before the UTI diagnosis & & \\
Hospitalization & $7.9 \%(3 / 38)$ & $(0 / 65)$ \\
Surgery & $(0 / 38)$ & - \\
Ureteral stent or urinary & $(0 / 38)$ & - \\
catheter & & $0(0 / 65)$ \\
Antibiotic therapy & $39.5 \%(15 / 38)$ & \\
\hline
\end{tabular}

Note: *Eight KTP information was missed.

acid $(58.3 \%)$ followed by ofloxacin $(47.7 \%)$, while the most effective antibiotic was carbapenems (100\%), amikacin $(100 \%)$, and nitrofurantoin $(89.2 \%)$. The frequencies of ESBL-positive strains were 31.5\% (35/111), out of which $20(43.5 \%)$ and $15(23.1 \%)$ strain isolated from KTP and control group, respectively. Moreover, the MDR phenotype was found in $71.7 \%$ and $41.5 \%$ of UPEC strain obtained from KTP and control group, respectively. Statistical analysis showed that resistance rate against cefepime, cefixim, trimethoprim/sulfamethoxazole, norfloxacin, and nalidixic acid were significantly higher among KTP than CA-UTI. Moreover, resistance rates of norfloxacin, piperacillin-tazobactam, ceftazidim, ofloxacin, ciprofloxacin, cefixim, and cefepime in ESBLpositive isolates were found to be higher than ESBLnegative isolates in KTP, while, resistance to cefoxitin, ceftazidim, cefixim, ciprofloxacin, ofloxacin, norfloxacin, and gentamicin ESBL-producing isolates in the control group was significantly higher than in non-ESBL producers $(\mathrm{P}, 0.001)$. The full results of antibiotic susceptibility pattern and comparison between ESBL-negative and ESBL-positive between two groups are shown in Tables 2 and 3. 


\begin{tabular}{|c|c|c|c|c|c|c|}
\hline \multicolumn{2}{|c|}{ 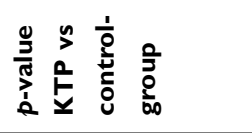 } & గ̂n & กี ฮี & $\begin{array}{l}\overline{8} \\
0 \\
\text { vi }\end{array}$ & 숭 웅 & 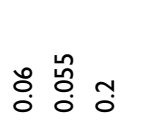 \\
\hline \multicolumn{2}{|c|}{$\dot{\frac{\mathscr{g}}{\pi}}$} & 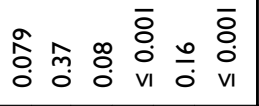 & & $\stackrel{m}{0}$ & 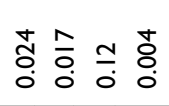 & 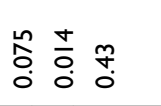 \\
\hline \multirow{2}{*}{ 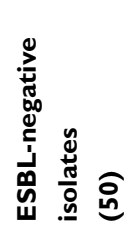 } & $n$ & 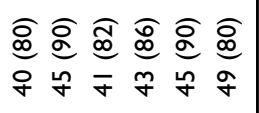 & $\begin{array}{l}\widehat{o} \stackrel{0}{\circ} \\
\text { o } \\
\text { 응 }\end{array}$ & $\begin{array}{l}\underset{0}{0} \\
\substack{\infty \\
\sim}\end{array}$ & 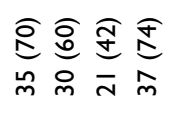 & 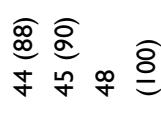 \\
\hline & $\propto$ & 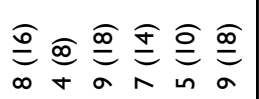 & 00 & $\begin{array}{l}\widehat{\widehat{\Phi}} \\
\bar{N}\end{array}$ & 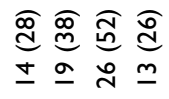 & 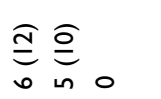 \\
\hline \multirow{2}{*}{ 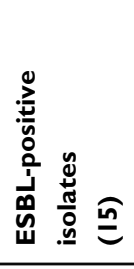 } & $n$ & 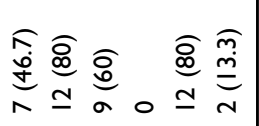 & 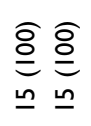 & $\underset{0}{\stackrel{9}{9}}$ & 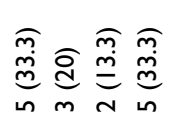 & 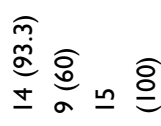 \\
\hline & $\propto$ & 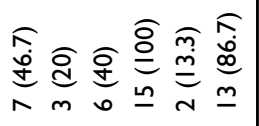 & 00 & $\begin{array}{l}\widehat{\alpha} \\
\text { o } \\
\sigma\end{array}$ & 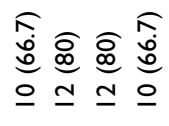 & $0 \stackrel{9}{0}_{0}$ \\
\hline \multicolumn{2}{|l|}{ 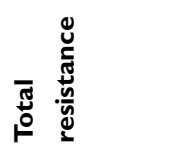 } & 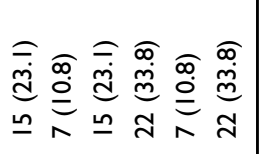 & 00 & $\begin{array}{l}\text { त̧ } \\
\text { jo } \\
0 \\
0\end{array}$ & 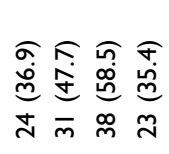 & 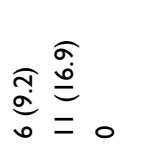 \\
\hline \multicolumn{2}{|c|}{ i } & 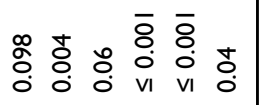 & & ò & 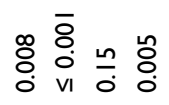 & $\stackrel{ \pm}{0}$ స్ \\
\hline \multirow{2}{*}{ 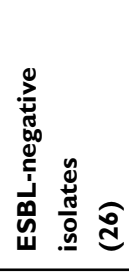 } & $n$ & 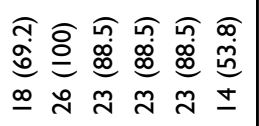 & 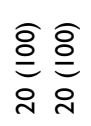 & 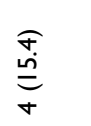 & 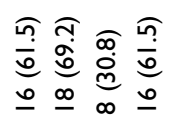 & 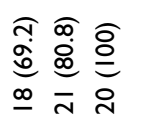 \\
\hline & $\propto$ & 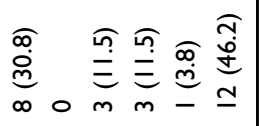 & 00 & 交 & 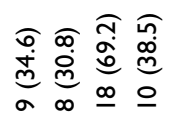 & 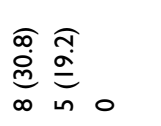 \\
\hline \multirow{2}{*}{ 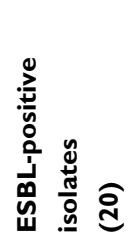 } & $n$ & 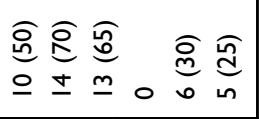 & 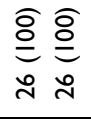 & 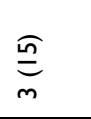 & 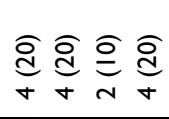 & 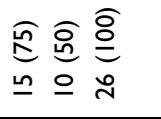 \\
\hline & $\propto$ & 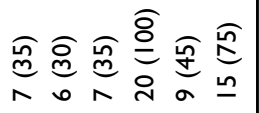 & 00 & 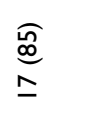 & 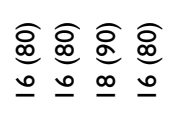 & 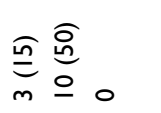 \\
\hline \multicolumn{2}{|l|}{ 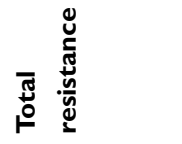 } & 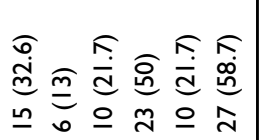 & 00 & $\begin{array}{l}\widehat{\infty} \\
\stackrel{+}{\infty} \\
\infty \\
\stackrel{\infty}{m}\end{array}$ & 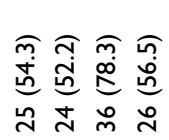 & 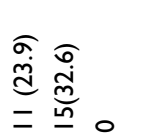 \\
\hline \multicolumn{2}{|c|}{ } & 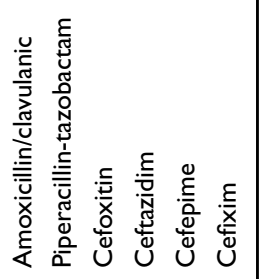 & 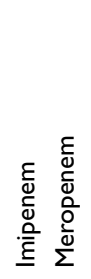 & 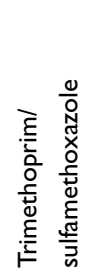 & 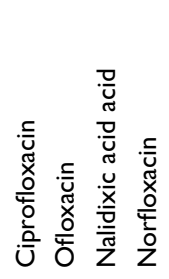 & 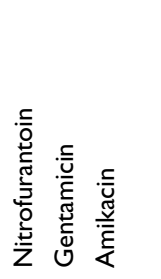 \\
\hline \multicolumn{2}{|c|}{ 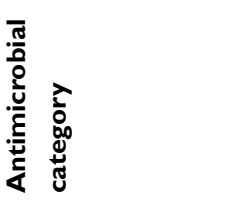 } & 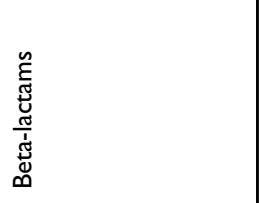 & 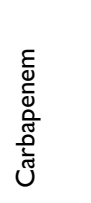 & 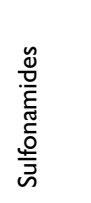 & $\begin{array}{l}\mathscr{ٌ} \\
\stackrel{0}{0} \\
\frac{0}{0} \\
\stackrel{\bar{\Xi}}{0} \\
0\end{array}$ & 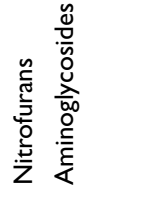 \\
\hline
\end{tabular}




\begin{tabular}{|c|c|c|c|c|c|c|c|c|}
\hline \multicolumn{3}{|c|}{ 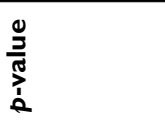 } & 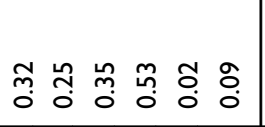 & กี ธี ธี & రิ & స్ & $\stackrel{m}{0}$ & 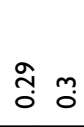 \\
\hline \multirow{4}{*}{ 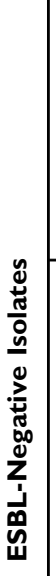 } & \multirow{2}{*}{ 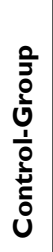 } & $n$ & 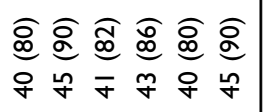 & 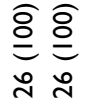 & $\begin{array}{l}\widehat{0} \\
\stackrel{0}{0} \\
\stackrel{\infty}{\infty}\end{array}$ & 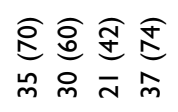 & $\begin{array}{l}\widehat{\mathscr{D}} \\
\stackrel{0}{0} \\
\bar{f}\end{array}$ & 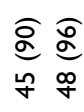 \\
\hline & & $\boldsymbol{x}$ & 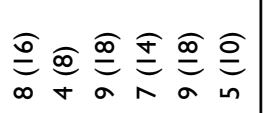 & 00 & $\begin{array}{l}\widehat{\mathcal{I}} \\
\bar{N}\end{array}$ & 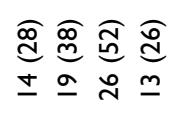 & $\underset{0}{\stackrel{\widehat{\Xi}}{\Xi}}$ & $\underbrace{\widehat{o}}_{n} \underset{N}{\mathbb{f}}$ \\
\hline & & $n$ & 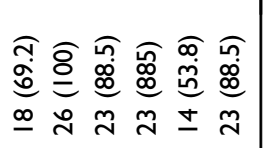 & 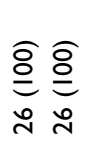 & 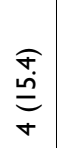 & 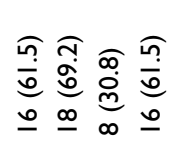 & $\begin{array}{l}\widehat{\widehat{a}} \\
\hat{\sigma} \\
\underline{\infty}\end{array}$ & 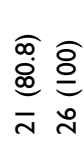 \\
\hline & & $\propto$ & 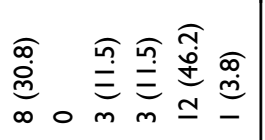 & 00 & 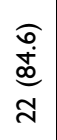 & 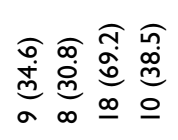 & $\begin{array}{l}\widehat{\infty} \\
\stackrel{0}{0} \\
\stackrel{\infty}{\infty}\end{array}$ & 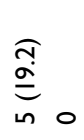 \\
\hline \multicolumn{3}{|c|}{ 突 } & 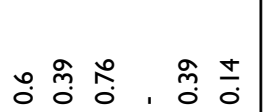 & 1 1 & $\overline{0}$ & mo & $\stackrel{\sim}{\sim}$ & นn \\
\hline \multirow{4}{*}{ 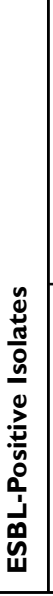 } & \multirow[t]{2}{*}{ 气े } & $n$ & 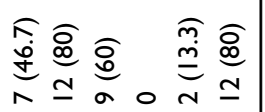 & 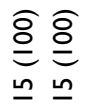 & $\underset{\sigma}{\mathfrak{q}}$ & 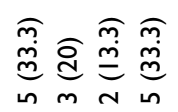 & 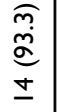 & 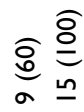 \\
\hline & & $\propto$ & 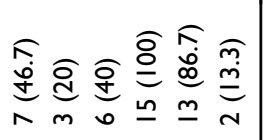 & 00 & $\begin{array}{l}\sigma \\
o \\
a\end{array}$ & 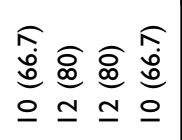 & 0 & $\underbrace{\frac{O}{9}}_{0}$ \\
\hline & & n & 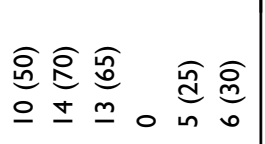 & 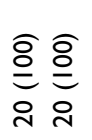 & 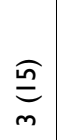 & 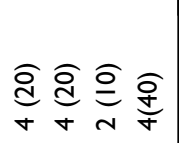 & $\begin{array}{l}\underline{\underline{n}} \\
\underline{\underline{n}}\end{array}$ & $\begin{array}{l}\text { ô } \\
\text { 응 } \\
\text { 응 }\end{array}$ \\
\hline & 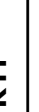 & $\propto$ & 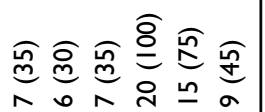 & 00 & $\begin{array}{l}\widehat{\nwarrow} \\
\stackrel{\infty}{\infty} \\
\simeq\end{array}$ & 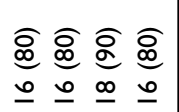 & 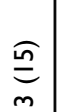 & 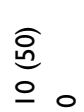 \\
\hline \multicolumn{3}{|c|}{ 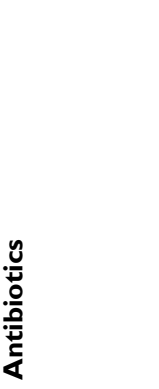 } & 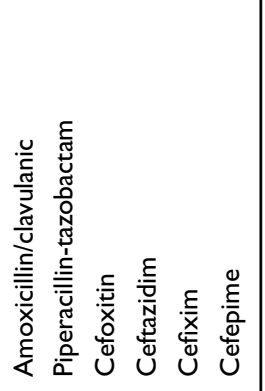 & 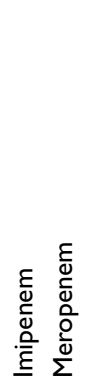 & 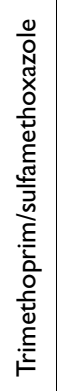 & 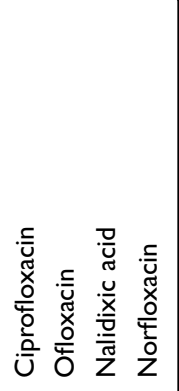 & 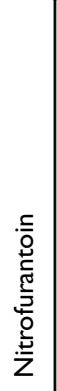 & 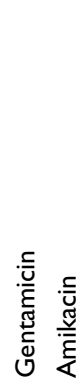 \\
\hline \multicolumn{3}{|c|}{ ț } & 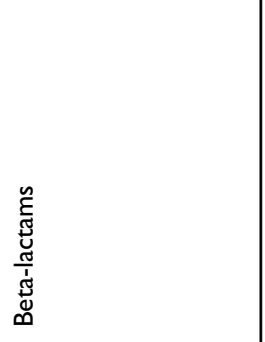 & 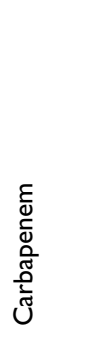 & 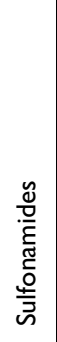 & $\begin{array}{l}\mathscr{y} \\
\stackrel{0}{0} \\
\frac{0}{0} \\
\stackrel{\bar{\Xi}}{0} \\
0\end{array}$ & 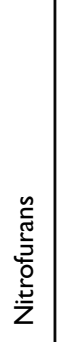 & 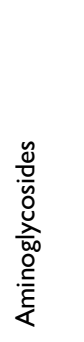 \\
\hline
\end{tabular}


The molecular results revealed that $53.2 \%$ (59/111), $45 \%(50 / 111)$, and $5.4 \%(6 / 111)$ of isolates harbored $b l a_{\mathrm{CTX}-\mathrm{M}}, \quad b l a_{\mathrm{TEM}}$ and $b l a_{\mathrm{SHV}}$ genes, respectively (Table 4). Also, 3 (2.7\%) of the isolates were found positive for all ESBL genes. Out of 35 ESBL-positive isolates (20 from KTP and 15 from control group), 29 isolates $(82.9 \%)$ harbored bla $_{\text {CTX-M followed by } 14(40 \%)}$ $b a_{\mathrm{TEM}}$, and $1(2.9 \%) b_{\text {ShV }}$. Of the studied resistance genes, $b l a_{\mathrm{CTX}-\mathrm{M}}$ and $b l a_{\mathrm{TEM}}$ genes were significantly higher among KTP than the control group, while bla $a_{\text {CTX-M }}$ were found significantly higher in ESBLpositive isolates in comparison with ESBL-negative isolates (Table 4). Moreover, 29 (26.1\%) isolates had both $b l a_{\mathrm{CTX}-\mathrm{M}}$ and $b l a_{\mathrm{TEM}}$, simultaneously.

\section{Discussion}

UTI is the common complication in KTPs. It is considered a particular risk factor for graft loss and patient death in kidney recipients, and it has seen in the first few weeks following transplantation. ${ }^{20}$ Gram-negative bacteria are the leading cause of $70 \%$ of UTIs, leading to renal failure of which, UPEC is the most common etiology. Furthermore, the extensive use of antibiotics in human medicine as treatment, prevention, and prophylactic is associated with the increasing emergence of MDR and ESBL-producing strains. ${ }^{21}$ Thus, it needs more attention to the prevention of UTI and its consequences in KTPs. ${ }^{22}$ Here, we investigated the antibiotic resistance pattern and ESBL production in a collection of UPEC obtained from KTPs and compared them to the control group. Physicians can use these regional antibiotic resistance rates to improve antimicrobial stewardship programs and alter antibiotic administration in these patients.

In the present study, $31.5 \%$ of the isolates were ESBL producers. According to our findings, ESBL-producing strains were significantly more frequent in KTPs compared to the control group. ( $43.5 \%$ vs $23.1 \%, P=0.021$ ).

In addition, in KTPs, the risk of UTIs provided by ESBL-producing isolates was 2.56 times (95\% CI 1.12 5.82) more than in the control group patients. These results highlight the importance of the presence and emergence of ESBL-producing isolates in these infections. According to the literature review, the high frequency of ESBL-positive as well as prophylactic antimicrobial therapy, and diabetes mellitus accounts for the potential risk factors for UTIs in KTPs. ${ }^{14,23}$ Furthermore, the high level of antibiotic resistance and high ESBL-producing isolates may show that KTPs are more exposed to high antibiotic selective

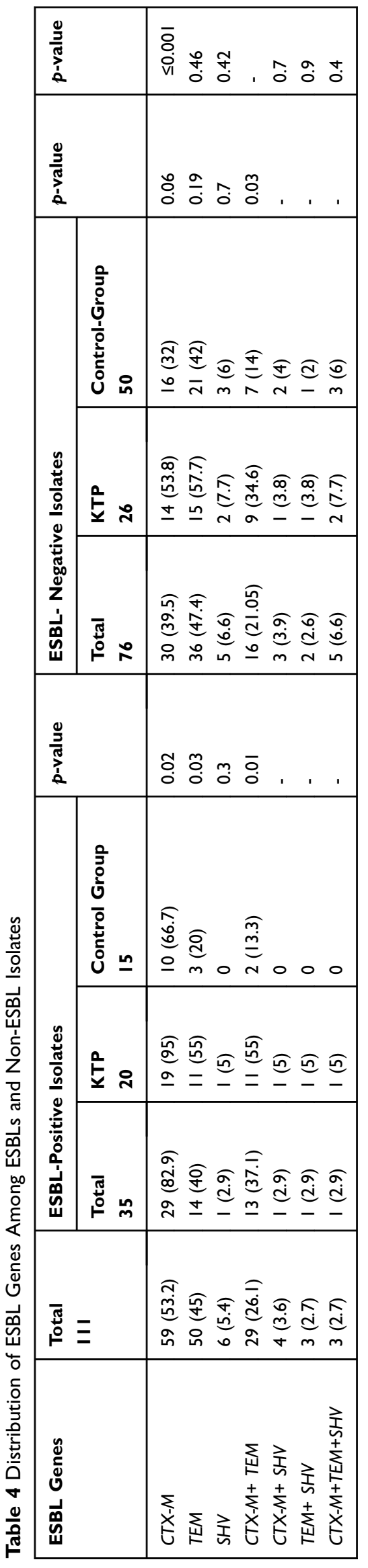


pressure and excessive use of antibiotics like thirdgeneration cephalosporins before and after transplantation. Various reports of ESBL-producing UPEC have been described from all parts of the world in KTPs. A lower frequency of ESBL-positive isolates has been reported in France $(10.9 \%),{ }^{24}$ Spain $(25.7 \%),{ }^{1}$ Canada (10.7\%), and the United States $(3.4 \%){ }^{25}$ A recent meta-analysis of the literature has reported that the incidence of the UTI caused by ESBL-producing Enterobacteriaceae in KTPs is $10 \%$. In accordance with our results, one study in northwestern of Iran reported that $40.6 \%$ of the UPEC isolates were ESBL producers. ${ }^{22}$

The incidence of ESBL-producing isolates among community-acquired UPEC isolates detected in this study $(23.1 \%)$ was higher than that reported among strains isolated in Brazil (7.1\%), ${ }^{26}$ Algeria (9\%), ${ }^{27}$ the United States $(7 \%$ to $15 \%),{ }^{28}$ and Turkey $(11 \%),{ }^{5}$ and it was lower than those reported in $\operatorname{Iran}(32 \%){ }^{29}(29.6 \%)^{30}$ and $(41 \%){ }^{31}$

In addition to increasing antibiotic resistance in KTPs, several factors such as intolerance of patients to some antibiotics and drug toxicity, and standard and appropriate antibiotic therapy for UPEC in KTPs are regarded as one of the complicated challenges. ${ }^{32}$ According to antibiotic susceptibility pattern, high resistance to trimethoprim/sulfamethoxazole and quinolones, as well as high effectiveness of carbapenems, amikacin, and nitrofurantoin were reported in KTPs. Moreover, relatively similar results were seen in the control group.

According to previous reports, third-generation cephalosporins and trimethoprim-sulfamethoxazole can be used as prophylaxis to reduce the incidence of UTI. ${ }^{3}$ However, based on our results showing high-level resistance against trimethoprim-sulfamethoxazole, the use of this antibiotic may not be as effective as prophylaxis.

Although another common treatment option is the use of quinolones for UTIs in KTPs, there have been increasing reports of decrease in susceptibility to quinolones. This increase is commonly associated with ESBL production. ${ }^{2,4,33}$ Compared to ESBL-negative isolates, ESBL-producing isolates were associated with high-level resistance to norfloxacin, piperacillin-tazobactam, ceftazidime, ofloxacin, ciprofloxacin, cefixime, and cefepime ( $\mathrm{P}$-value $<0.05)$. This finding is one of the most worrisome aspects of ESBL-positive bacteria.

This pattern of antibiotic resistance was very close to the finding obtained by Mohammadzadeh et al, ${ }^{32}$ Kashef Nejad et al, ${ }^{22}$ and Azap et $\mathrm{al}^{4}$ reporting a high level of resistance to trimethoprim/sulfamethoxazole and quinolones and also carbapenems had the least therapeutic effect. Commonly, in KTP, co-trimoxazole with $480 \mathrm{mg}$ daily is used for 6 months after transplantation. In a metaanalysis study conducted by Moghaddam et al, antibiotic susceptibility pattern of UPEC isolates among Iranian KTP patients was investigated. Similar to our results, the results indicated that cotrimoxazole, nalidixic acid, cefixime, and ceftazidime had a high level of antibiotic

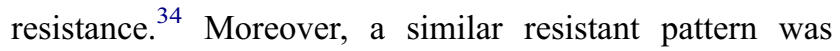
also reported by Shokouhi Mostafavi et $\mathrm{al}^{35}$ and Farajzadah Sheikh et $\mathrm{al}^{8}$ in Iran.

In our region, based on the literature review, the resistance rate of the isolates was high to fluoroquinolones, sulfonamides, and third-generation cephalosporins. This high resistance rate may be due to the extensive and routine use of these agents in treatment patients with UTI. ${ }^{8,22,30,32,35}$

Similar to our finding, a report from China established that among KTPs, all gram-negative bacteria like UPEC were susceptible to carbapenems (imipenem or meropenem) that might be related to its low usage in routine treatments for UTIs. In this regard, carbapenems such as imipenem and meropenem represent a good choice for serious UTIs. $^{22}$ Nevertheless, therapy with carbapenems should be implemented with caution, since the emergence of carbapenem-resistance tends to increase among Gramnegative organisms with ESBLs. ${ }^{36}$ Accordingly, our results suggest that nitrofurantoin and aminoglycoside can be used as drugs available for outpatient setting.

Molecular analysis of the three most prevalent ESBL genes (bla $a_{\mathrm{CTX}-\mathrm{M}}, b l a_{\mathrm{TEM}}$, and $\left.b l a_{\mathrm{SHV}}\right)$ showed that $b l a_{\mathrm{CTX}-\mathrm{M}}$ was the most common ESBL gene and following that $b l a_{\mathrm{TEM}}(45 \%)$ was higher among all UPEC isolates. Furthermore, a significant association was detected between the presence of bla $a_{\mathrm{CTX}-\mathrm{M}}$ and ESBL-positive strain $(P \leq 0.001)$; however, bla higher frequency in ESBL-negative strain $(P=0.46)$.

The considerable predominance of the $b l a_{\mathrm{CTX}-\mathrm{M}-15}$ allele may be owing to the outstanding ability of its gene products to hydrolyze aztreonam, cefotaxime, and ceftazidime. According to some previous studies, the $b l a_{\mathrm{CTX}-\mathrm{M}}$ is the most frequent beta-lactamase in UPEC isolates. ${ }^{27,31,37}$ This finding confirms the importance of the spread of bla $a_{\text {CтХ-M }}$ types in ESBL production.

Additionally, this report demonstrates that the presence of a $b l a_{\mathrm{CTX}-\mathrm{M}}$ gene in a bacterium may be an appropriate biomarker for high resistance to beta-lactam antibiotics, and it can be applied in protocols associated with testing routine antibiotic susceptibility. 
Furthermore, in this study, the presence of $b l a_{\text {CTX-M }}$ and $b l a_{\mathrm{TEM}}$ was significantly higher in KTP isolates than in the control group. In Portugal, Espinar et al investigated the prevalence of ESBL among KTPs. In this study, 25\%, $18.8 \%$, and $9.4 \%$ of isolates carried $b l a_{\mathrm{CTX}-\mathrm{M}}, b l a_{\mathrm{TEM}}$, and $b l a_{\text {SHV }}$, respectively. ${ }^{38}$ This finding was inconsistent with our results revealing $82.9 \%, 40 \%$, and $2.9 \%$ of isolates harbored $b l a_{\mathrm{CTX}-\mathrm{M}}, b l a_{\mathrm{TEM}}$, and $b l a_{\mathrm{SHV}}$, respectively. Based on our findings, the occurrence of $b l a_{\mathrm{SHV}}$ gene was lower than that reported in $\operatorname{Iran}^{31,37,39}$ and Portugal, ${ }^{38}$ but it was similar to that reported in Turkey. ${ }^{5}$ Despite the relative agreement on the higher incidence of ESBLs genes among UPECs, a discrepancy in the prevalence of ESBL producers was observed over different regions, which is probably due to differences in geographical regions, infection control policy, and sample source. The present study has some limitations that we should discuss. The first limitation of this study is the lack of complete background details of patient history. The second is the incomplete data on documentation of symptoms and physical exam.

\section{Conclusion}

In conclusion, this study demonstrated a high frequency of MDR and ESBLs isolates that most of them harbor $b l a_{\text {CTX-м }}$ in KTPs. Significant high co-resistance to different classes of antibiotics was reported from ESBLproducing UPEC in UTIs from KTPs, which remains a serious clinical challenge. Thus, this high level of resistance and high frequency of MDR and ESBLs isolates may alert us to adjust our strategy regarding empirical antibiotic therapy and screening susceptibility pattern for UTI cases among KTPs.

\section{Acknowledgments}

The authors would like to appreciate the Department of Microbiology of Isfahan University of Medical Sciences.

\section{Author Contributions}

All authors contributed to data analysis, drafting or revising the article, gave final approval of the version to be published, and agree to be accountable for all aspects of the work.

\section{Disclosure}

The authors report no conflicts of interest in this work.

\section{References}

1. Vidal E, Torre-Cisneros J, Blanes M, et al. Bacterial urinary tract infection after solid organ transplantation in the RESITRA cohort. Transpl Infect Dis. 2012;14:595-603. doi:10.1111/j.1399-3062.2012. 00744.x

2. Farajzadeh Sheikh A, Shahin M, Shokoohizadeh L, Halaji M, Shahcheraghi F, Ghanbari F. Molecular epidemiology of colistin-resistant Pseudomonas aeruginosa producing NDM-1 from hospitalized patients in Iran. Iran J Basic Med Sci. 2019;22:38-42. doi:10.22038/ijbms.2018.29264.7096

3. Rawat D, Nair D. Extended-spectrum $\beta$-lactamases in gram negative bacteria. J Glob Infect Dis. 2010;2:263-274. doi:10.4103/0974777X.68531

4. Azap OK, Arslan H, Serefhanoglu K, et al. Risk factors for extended-spectrum beta-lactamase positivity in uropathogenic Escherichia coli isolated from community-acquired urinary tract infections. Clin Microbiol Infect. 2010;16(2):147-151. doi:10.1111/ j.1469-0691.2009.02941.x

5. Azap O, Togan T, Yesilkaya A, Arslan H, Haberal M. Antimicrobial susceptibilities of uropathogen Escherichia coli in renal transplant recipients: dramatic increase in ciprofloxacin resistance. Transplant Proc. 2013;45(3):956-957. doi:10.1016/j.transproceed.2013.03.006

6. Paul R. State of the globe: rising antimicrobial resistance of pathogens in urinary tract infection. $J$ Glob Infect Dis. 2018;10 (3):117-118. doi:10.4103/jgid.jgid_104_17

7. Faghri J, Nouri S, Jalalifar S, Zalipoor M, Halaji M. Investigation of antimicrobial susceptibility, class I and II integrons among Pseudomonas aeruginosa isolates from hospitalized patients in Isfahan, Iran. BMC Res Notes. 2018;11(1):806. doi:10.1186/s13104018-3901-9

8. Farajzadah Sheikh A, Goodarzi H, Yadyad MJ, et al. Virulenceassociated genes and drug susceptibility patterns of uropathogenic Escherichia coli isolated from patients with urinary tract infection. Infect Drug Resist. 2019;12:2039-2047. doi:10.2147/IDR.S199764

9. Alevizakos M, Nasioudis D, Mylonakis E. Urinary tract infections caused by ESBL-producing Enterobacteriaceae in renal transplant recipients: a systematic review and meta-analysis. Transpl Infect Dis. 2017;19.

10. Machado E, Cantón R, Baquero F, et al. Integron content of extended-spectrum- $\beta$-lactamase-producing Escherichia coli Strains over 12 years in a single hospital in Madrid, Spain. Antimicrob Agents Chemother. 2005;49:1823. doi:10.1128/AAC.49.2.612-618.20 05

11. Bodro M, Sanclemente G, Lipperheide I, et al. Impact of antibiotic resistance on the development of recurrent and relapsing symptomatic urinary tract infection in kidney recipients. Am J Transplant. 2015;15:1021-1027. doi:10.1111/ajt.13075

12. Kaur M, Aggarwal A. Occurrence of the CTX-M, SHV and the TEM genes among the extended spectrum beta-lactamase producing isolates of enterobacteriaceae in a tertiary Care Hospital of North India. J Clin Diagn Res. 2013;7:642-645. doi:10.7860/JCDR/2013/5081. 2872

13. Ur Rahman S, Ali T, Ali I, Khan NA, Han B, Gao J. The growing genetic and functional diversity of extended spectrum beta-lactamases. Biomed Res Int. 2018;2018:9519718. doi:10.1155/ 2018/9519718

14. Hollyer I, Ison MG. The challenge of urinary tract infections in renal transplant recipients. Transpl Infect Dis. 2018;20:e12828. doi:10.11 11/tid. 12828

15. Jouini A, Vinue L, Slama KB, et al. Characterization of CTX-M and SHV extended-spectrum beta-lactamases and associated resistance genes in Escherichia coli strains of food samples in Tunisia. J Antimicrob Chemother. 2007;60:1137-1141. doi:10.1093/jac/dkm 316 
16. Wayne P. Clinical and Laboratory Standards Institute: Performance Standards for Antimicrobial Susceptibility Testing: Twenty-Fourth Informational Supplement, M100-S24. Clinical Laboratory Standards Institute (CLSI). 2014;34.

17. Magiorakos AP, Srinivasan A, Carey RB, et al. Multidrug-resistant, extensively drug-resistant and pandrug-resistant bacteria: an international expert proposal for interim standard definitions for acquired resistance. Clin Microbiol Infect. 2012;18:268-281. doi:10.1111/ j.1469-0691.2011.03570.x

18. Edelstein M, Pimkin M, Palagin I, Edelstein I, Stratchounski L. Prevalence and molecular epidemiology of CTX-M extended-spectrum beta-lactamase-producing Escherichia coli and Klebsiella pneumoniae in Russian hospitals. Antimicrob Agents Chemother. 2003;47:3724-3732. doi:10.1128/AAC.47.12.3724-3732.2003

19. Wiegand I, Geiss HK, Mack D, Sturenburg E, Seifert H. Detection of extended-spectrum beta-lactamases among Enterobacteriaceae by use of semiautomated microbiology systems and manual detection procedures. J Clin Microbiol. 2007. 45:1167-1174. doi:10.1128/JCM.01988-06

20. Shams SF, Eidgahi ES, Lotfi Z, et al. Urinary tract infections in kidney transplant recipients 1 (st) year after transplantation. $J$ Res Med Sci. 2017;22:20. doi:10.4103/1735-1995.200274

21. Li B, Webster TJ. Bacteria antibiotic resistance: new challenges and opportunities for implant-associated orthopedic infections. J Orthop Res. 2018;36:22-32. doi:10.1002/jor.23656

22. Kashef Nejad M, Jazani NH, Sharifi Y. Urinary tract infections among kidney transplant patients due to extended-spectrum beta-lactamase-producing bacteria. Microb Pathog. 2017;107:27 6-279. doi:10.1016/j.micpath.2017.03.046

23. Pinheiro H, Mituiassu A, Carminatti M, Braga A, Bastos M. Urinary tract infection caused by extended-spectrum beta-lactamase-producing bacteria in kidney transplant patients. In: Transplant Proc. Elsevier; 2010:486-487.

24. Pilmis B, Scemla A, Join-Lambert O, et al. ESBL-producing enterobacteriaceae-related urinary tract infections in kidney transplant recipients: incidence and risk factors for recurrence. Infect Dis. 2015;47(10):714-718. doi:10.3109/23744235.2015.1051107

25. Ramadas P, Rajendran PP, Krishnan P, et al. Extended-spectrum-betalactamase producing bacteria related urinary tract infection in renal transplant recipients and effect on allograft function. PLoS One. 2014;9(3):e91289. doi:10.1371/journal.pone.0091289

26. Goncalves LF, de Oliveira Martins-junior P, de Melo ABF, et al. Multidrug resistance dissemination by extended-spectrum beta-lactamase-producing Escherichia coli causing community-acquired urinary tract infection in the Central-Western Region, Brazil. J Glob Antimicrob Resist. 2016;6:1-4. doi:10.1016/j.jgar.2016.02.003

27. Nabti LZ, Sahli F, Radji N, et al. High prevalence of multidrug-resistant Escherichia coli in urine samples from inpatients and outpatients at a tertiary care hospital in Setif, Algeria. Microb Drug Resist. 2019;25:386-393. doi:10.1089/mdr.2018.0314

28. Zhu FH, Rodado MP, Asmar BI, Salimnia H, Thomas R, Abdel-Haq $\mathrm{N}$. Risk factors for community acquired urinary tract infections caused by extended spectrum beta-lactamase (ESBL) producing Escherichia coli in children: a case control study. Infect Dis (Lond). 2019;51:802-809. doi:10.1080/23744235.2019.1654127
29. Pourakbari B, Ferdosian F, Mahmoudi S, et al. Increase resistant rates and ESBL production between E. coli isolates causing urinary tract infection in young patients from Iran. Braz $J$ Microbiol. 2012;43:766-769. doi:10.1590/S1517-83822012000200041

30. Shahbazi S, Asadi Karam MR, Habibi M, Talebi A, Bouzari S. Distribution of extended-spectrum beta-lactam, quinolone and carbapenem resistance genes, and genetic diversity among uropathogenic Escherichia coli isolates in Tehran, Iran. J Glob Antimicrob Resist. 2018;14:118-125. doi:10.1016/j.jgar.2018.03.006

31. Hashemizadeh Z, Kalantar-Neyestanaki D, Mansouri S. Clonal relationships, antimicrobial susceptibilities, and molecular characterization of extended-spectrum beta-lactamase-producing Escherichia coli isolates from urinary tract infections and fecal samples in Southeast Iran. Rev Soc Bras Med Trop. 2018;51:44-51. doi:10.1590/00378682-0080-2017

32. Mohammadzadeh M, Tavakoli M, Yaslianifard S, Asadi E, Golmohammadi R, Mirnejad R. Genetic diversity and antibiotic susceptibility of uropathogenic Escherichia coli isolates from kidney transplant recipients. Infect Drug Resist. 2019;12:1795-1803.

33. Fasugba O, Gardner A, Mitchell BG, Mnatzaganian G. Ciprofloxacin resistance in community- and hospital-acquired Escherichia coli urinary tract infections: a systematic review and meta-analysis of observational studies. BMC Infect Dis. 2015;15(1):545. doi:10.1186/ s12879-015-1282-4

34. Shapouri Moghaddam A, Arfaatabar M, Tavakol Afshari J, Shakerimoghaddam A, Mohammadzamani Z, Khaledi A. Prevalence and antimicrobial resistance of bacterial uropathogens isolated from iranian kidney transplant recipients: a systematic review and meta-analysis. Iran J Public Health. 2019;48:2165-2176.

35. Shokouhi Mostafavi SK, Najar-Peerayeh S, Mohabbati Mobarez A, Kardoust Parizi M. Serogroup distribution, diversity of exotoxin gene profiles, and phylogenetic grouping of CTX-M-1- producing uropathogenic Escherichia coli. Comp Immunol Microbiol Infect Dis. 2019;65:148-153. doi:10.1016/j.cimid.2019.05.003

36. Shahraki-Zahedani S, Moghadampour M, Bokaeian M, AnsariMoghaddam A. Prevalence of CTX-M-8 and CTX-M-15 type extended-spectrum beta-lactamases between Klebsiella pneumoniae spp. isolated from Zahedan, Southeast Iran. $J$ Chemother. 2016;28:343-345. doi:10.1179/1973947815Y.0000000008

37. Zeighami H, Haghi F, Hajiahmadi F. Molecular characterization of integrons in clinical isolates of betalactamase-producing Escherichia coli and Klebsiella pneumoniae in Iran. $J$ Chemother. 2015;27:145-151. doi:10.1179/1973947814Y.0000000180

38. Espinar MJ, Miranda IM, Costa-de-Oliveira S, Rocha R, Rodrigues AG, Pina-Vaz C. Urinary tract infections in kidney transplant patients due to escherichia coli and klebsiella pneumoniae-producing extended-spectrum beta-lactamases: risk factors and molecular epidemiology. PLoS One. 2015;10:e134737. doi:10.1371/journal.pone. 0134737

39. Namaei MH, Yousefi M, Ziaee M, et al. First report of prevalence of CTX-M-15-producing Escherichia coli O25b/ST131 from Iran. Microb Drug Resist. 2017;23:879-884. doi:10.1089/mdr.2016.0272
Infection and Drug Resistance

\section{Publish your work in this journal}

Infection and Drug Resistance is an international, peer-reviewed openaccess journal that focuses on the optimal treatment of infection (bacterial, fungal and viral) and the development and institution of preventive strategies to minimize the development and spread of resistance. The journal is specifically concerned with the epidemiology of antibiotic resistance and the mechanisms of resistance development and diffusion in both hospitals and the community. The manuscript management system is completely online and includes a very quick and fair peerreview system, which is all easy to use. Visit http://www.dovepress.com/ testimonials.php to read real quotes from published authors. 